Journal of Accident and Emergency Medicine 1995 12, 302-303

Short reports

\section{Aspirin and suspected myocardial infarction: a life-saving treatment we too often forget}

Recent studies suggest that patients with myocardial infarction or unstable angina may not receive lifesaving treatment. ${ }^{1-3}$ Aspirin reduces mortality in these patients. ${ }^{4}$ This study assessed the impact of these studies on general practitioners (GPs) and, because many patients with ischaemic chest pain present directly to them, on accident and emergency (A\&E) staff.

Two 4-week periods (20 February to 9 March 1994 - the date of publication of references $1,2,3$ and 5 with an accompanying editorial - and 20 March to 16 April) were assessed retrospectively to determine whether the practice of doctors was altered by the publication of reports which highlighted inadequacies in the management of patients with ischaemic cardiac pain. The A\&E patients admitted to the coronary care unit via the A\&E department were reviewed; it was noted whether aspirin was prescribed.

Patients were included in the audit if they were not taking regular aspirin and were admitted to coronary care with ischaemic chest pain. Patients were excluded from analysis if there were a documented contraindication to aspirin. The two periods were compared using Fischer's exact test and the $\chi^{2}$ test.

A total of 78 patients, who were not already receiving aspirin, were admitted in the study period. Four $(5 \%)$ were excluded because aspirin was contraindicated. The notes of eight patients could not be traced. The management of 66 patients was assessed (39 in the first period, 27 in the second). Fourteen of these $(21 \%)$ did not receive aspirin before admission to the coronary care unit.

In the first period 12 patients had been seen by their GP: none had received aspirin. In the second period aspirin had been given to two of the 10 patients who were seen by their GP $(P=0.195$, Fisher's exact test).

In the first period, 39 patients who had not already received aspirin were seen by A\&E staff: $19(49 \%)$ received aspirin. Aspirin was given to 10 of the $24(42 \%)$ eligible patients in the second period (one patient was given aspirin by a paramedic). This difference is not significant $\left(\chi^{2}=\right.$ 0.2974).

Coronary care staff prescribed aspirin to 11 of the $20(55 \%)$ eligible patients in the first study period and nine of the $14(64 \%)$ in the second; not significant $\left(\chi^{2}=0.2931\right)$.

The data to support the use of aspirin in this setting are compelling; aspirin reduces mortality in acute myocardial infarction. ${ }^{6}$ The estimated relative risk reduction is $23 \%{ }^{4}$ (from 11.7 to $9.3 \%$ ), equivalent to one less death for 42 treated patients. The risk of an acute myocardial infarction, stroke or vascular death in patients with unstable angina is reduced from 14.1 to $9.1 \%$, equivalent to one fewer adverse event for 20 treated patients. ${ }^{4}$

When discussing the management of patients with acute myocardial infarction emphasis is often given to minimizing the delay in thrombolysis. Aspirin treatment should not be forgotten; it is less often contraindicated than thrombolysis, is less likely to cause adverse effects and has superior cost-benefit and risk-benefit ratios. ${ }^{7}$ Moreover, aspirin may act synergistically with thrombolytics; ${ }^{8}$ delays in aspirin administration may reduce the benefit of thrombolysis.

Some patients with suspected myocardial infarction receive suboptimal care. Fourteen patients ( $21 \%$ of those eligible for aspirin treatment) were admitted to the coronary care unit without receiving aspirin beforehand.

Although it has been reported that use of aspirin in acute myocardial infarction became more common after the publication of ISIS- $2,{ }^{9}$ the present study suggests that the publication of guidelines ${ }^{5}$ without associated education and audit does not alter doctors' behaviour. Local protocols for the management of patients with ischaemic cardiac pain should be drawn up for all staff involved in their care, so that patients receive prompt treatment with effective drugs. Adherence to these protocols should then be audited.

\section{ACKNOWLEDGEMENT}

I thank Mr J.P. Sloan, Consultant in accident and emergency medicine, for his permission to report patients attending this department.

\section{REFERENCES}

1. Moher M. \& Johnson N. (1994) Use of aspirin by general practitioners in suspected myocardial infarction. British Medical Journal 308, 760.

2. Wyllie H.R. \& Dunn F.G. (1994) Prehospital opiate and aspirin administration in patients with suspected myocardial infarction. British Medical Journal 308, 760-761.

(c) 1995 Blackwell Science Ltd. 
3. Moher M., Moher D. \& Havelock P. (1994) Survey of whether general practitioners carry aspirin in their doctor's bag. British Medical Journal 308, 761-762.

4. Anti-platelet Trialists' Collaboration (1994) Collaborative overview of randomised trials on antiplatelet therapy: prevention of death, myocardial infarction and stroke by prolonged antiplatelet therapy in various categories of patients. British Medical Journal 308, 81-106.

5. Weston C.F.M., Penny W.J. \& Julian D.G. on behalf of the British Heart Foundation Working Group (1994). Guidelines for the early management of patients with myocardial infarction. British Medical Journal 308, 767-771.

6. Lau J., Antman E.M., Jiminez-Silva J., Kupelnick B., Mosteller F. \& Chalmers T.C. (1992) Cumulative metaanalysis trials for myocardial infarction. New England Journal of Medicine 327, 248-254.

7. Hennekens C.H., Jonas M.A. \& Buring J.E. (1994) The benefits of aspirin in acute myocardial infarction. Still a well kept secret in the USA. Archives of Internal Medicine 154, 37-39.

8. Basinski A. \& Naylor C.D. (1991) Aspirin and fibrinolysis in acute myocardial infarction: meta-analytic evidence for synergy. Journal of Clinical Epidemiology 44, 1085-1096.

9. Lamas G.A., Pfeffer M.A., Hamm P., Wertheimer J., Rouleau J.-L. \& Braunwald E. (1992) Do the results of randomised clinical trials of cardio-vascular drugs influence medical practice? New England Journal of Medicine 327, 241-247.

\section{R.D. HARDERN}

Accident and Emergency Department, The General Infirmary, Great George Street, Leeds LS1 3EX, UK

\section{Removal of fish bones from the throat}

Patients with fish bones impacted in their throats commonly present to accident and emergency (A\&E) departments and if the bones are not removed quickly they can produce serious sequelae. A plain radiograph may identify some bones, but this technique is unreliable as many commonly eaten fish (e.g. mackerel, trout and salmon) have radiolucent skeletons.' Most retained fish bones are visible on initial examination of the oropharynx ${ }^{2}$ and are usually lodged in the base of the tongue, tonsils or posterior pharyngeal wall.

A simple method is described to remove fish bones impacted in the pharynx using instruments that are invariably available in every A\&E department.

With the patient seated, the operator inserts the Magill laryngoscope as shown in Fig. 1. This gives an excellent view of the affected area and keeps the tongue retracted, leaving the operator's other hand free to remove the bone using Magill forceps.

\section{REFERENCES}

1. Carr J. (1987) Radiology of fish bone foreign bodies in the neck. Joumal of Laryngology and Otology 101, 407-410.

2. Knight L.C \& Lesser T.H.J. (1989) Fish bones in the throat. Archives of Emergency Medicine 6, 13-16.

\section{B. NATHAN, S. PREMACHANDRAN \& K. HASHEMI}

Accident and Emergency Department, Mayday University Hospital, Croydon, Surrey CR7 TYE, UK

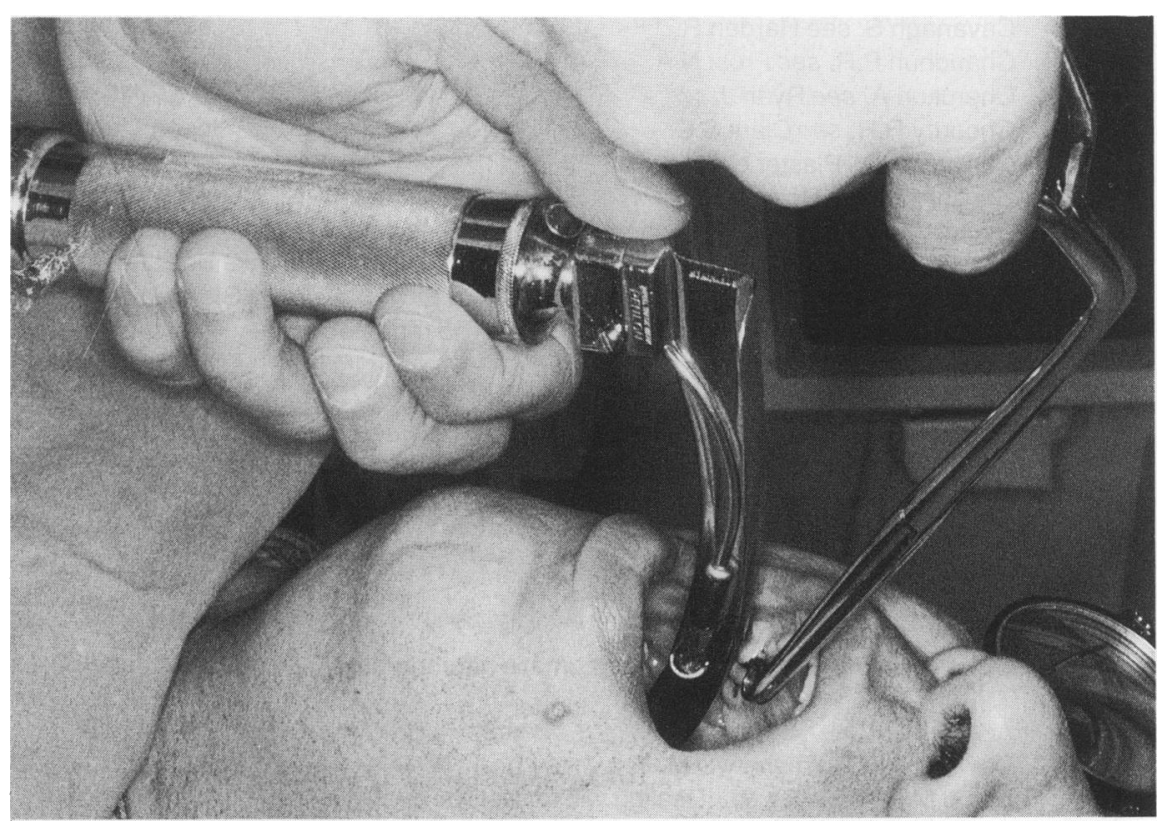

Fig. 1. Removal of fish bones from a patient's throat using a Magill lanyngoscope and Magill forceps. 